\title{
LA TRANSCOMPLEJIDAD. UNA VISIÓN EPISTEMOLÓGICA DEL SIGLO XXI
}

\author{
Óscar Fernández Galíndez \\ Universidad Nacional Experimental Simón Rodríguez, Venezuela
}

Resumen.- La transcomplejidad vista ésta a través del ojo del ciberpensamiento y mediado a su vez por la educación cuántica nos ofrece una revisión paradigmática que sugiere la integración neokantiana del sentir/pensar/hacer a través la crítica a la ciencia a la ética y al arte, no como instancias separadas sino complementarias. Que se tejen en y/o a través de la red de redes. Así pues pensar transcomplejamente es sentir y hacer coherente y congruentemente con y para la vida.

Palabras-clave: transcomplejidad, educación cuántica, ciberpensamiento, ciberética, ciberestética

\section{The transcomplexity. An epistemological vision of the 21 st century}

Abstract.- The seen transcomplejidad this one across the eye of the cyberthought and happened in turn by the quantum education it offers us a paradigmatic review that suggests the integration neokantian of to be sorry / to think / do to slant the critique to the science to the ethics and to the art, not as separated but complementary instances. That are woven in and / or across the network of networks. This way so to think transcomplextly is to be sorry and to do coherent and coherently with and for the life.

Keywords: transcomplejidad, quantum education, cyberthought, cyberethics, cyberaesthetics

\section{INTRODUCCIÓN}

El pensar sobre la transcomplejidad nos conduce en primer lugar a tratar de entender su origen etimológico en el que complejidad se refiere a lo que se tejel junto, lo que se relaciona, lo que interactúa. En términos propios de Pascal sería igual a decir que todo tiene que ver con todo. Sinembargo desde esta perspectiva, cabría decir que si bien todo se relaciona de algún modo con todo, esto no significa que todo se

1 Debemos destacar aquí que en este texto el término tejido es equivalente al término red, y en consecuencia también todas sus derivaciones. 
conecta de cualquier forma con todo. Porque para que algo se teja con algo más, debe existir armonía. Debe existir lo que podríamos denominar lógicas de codependencia. En términos de Jacob von Üexkull, debe existir un dúo en la sinfonía de la vida. Él nos decía que para que el dúo abeja/flor se diera, la flor debía ser apícola y la abeja debía ser floral.

Por otra parte tenemos al prefijo trans, que se antepone al término complejidad. "Trans"2 significa más allá de, pero también más acá y entre. La transdisciplinariedad por ejemplo, se encuentra entremezclada a través de la complejidad. Para Nicolescu; los tres pilares metodológicos de la transdisciplinariedad son: la lógica del tercer incluido, los niveles de la realidad y la complejidad. De esta forma complejidad y transdisciplinariedad se entretejen a tal punto que casi no sabemos cuál es cual.

Por lo tanto podríamos decir que la transcomplejidad debería significar lo que se encuentra más allá del tejido de la vida, pero también lo que está entre lo vivo y lo no vivo y sobre todo lo que se encuentra en medio de cada tejido. Tejiendo sentidos, tejiendo relaciones, tejiendo realidades, tejiendo posibilidades. Y estas posibilidades se dan tanto a nivel de los organismos individuales como a nivel de las poblaciones y comunidades de estos. En el caso nuestro también podemos hablar desde aquí de la transcomplejidad, vista ésta en y/o a través del corpus societal.

En el orden metodológico la transcomplejidad además de incorporar la mirada propia de la transdisciplinariedad que considera en la praxis a la incorporación de lo cuantitativo con lo cualitativo agregamos además lo que algunos han denominado como metodología espiritual de allí las propuestas hechas por Martos a través de la educación cuántica y las hechas por mí a través de la metodología neurofenomenológica biohermenéutica ${ }^{3}$

En resumen, transcomplejidad a juicio de este humilde escritor, es lo que emerge de las relaciones entre un tejido y otro pero también es el tejido mismo con cada una de sus partes que interactúan sistémicamente en esta recursiva y a la vez espirílica red relacional que llamamos vida.

2 Sería interesante destacar que tanto los prefijos trans, meta, pos y post. En algunos casos son utilizados para significar más o menos lo mismo, y en otros no. Tal es el caso del transhumanismo que se ha vuelto una especie de tecnoreligión y el posthumanismo un cuestionamiento crítico a la misma.

3 FERNÁNDEZ, O. (2018) Metodología Neurofenomenológica-Biohermenéutica. Centro Nacional de Desarrollo e Investigación en Tecnologías Libres (CENDITEL) Revista Electrónica Conocimiento Libre y Licenciamiento (CLIC), Mérida - Venezuela ISSN: 22447423

EMUI_EuroMed University | ISSN 1578-6730 · Nomads. Mediterranean Perspectives | ISSN 1889-7231 (C) 1999, Román Reyes, Fundador y Director · (C) 2016, Progetto Pier Paolo Pasolini 
POSTHUMANISMO TRANSRACIONAL A TRAVÉS DE LA ATEMPORALIDAD/AESPACIALIDAD DEL CIBERPENSAMIENTO, EL CIBERARTE $Y$ LA CIBERÉTICA

La mirada posthumana que sugiere un replanteamiento del humanismo expresado por medio del acercamiento hombre-máquina, máquinahombre e incluso máquina-máquina, nos sumerge en una paradoja tetrádica que involucra a la relación: NATURALEZA, HOMBRE, CULTURA, OBJETOS. Y nos invita a hacernos en principio la siguiente pregunta: $\dot{z} L O S$ hombres hacen los objetos o los objetos hacen al hombre?4

Según sea la respuesta será la perspectiva desde y/o a través de la cual, nos posicionemos ante esa cosa casi inexistente que llamamos realidad.

En esta ensalada subjetivo-simbólica, los ingredientes de dicha dieta racional y/o espiritual, definirán el sentido interpretativo de quien pretenda enunciar alguna construcción discursiva. Es así como hasta el mismo lenguaje se convierte en sujeto de cuestionamiento, dado que éste también deriva de la misma lógica-simbólica que lo limita.

Así pues podremos por ejemplo vislumbrar muchas lecturas fatalistas postapocalípticas que muestren a un mundo casi destruido, contaminado y controlado por máquinas irónicamente más inteligentes que sus creadores. $U$ otro menos imaginado por los cientistas ficcionarios, ya que éste resulta menos atractivo y vende menos libros y/o películas. Desde esta óptica poco popular se plantea una convivencia pacífica y armónica con dichas máquinas o incluso un mundo en el que dichos artefactos provistos de inteligencia artificial, nos enseñen a ser mejores personas.

El ciberarte o ciberestética teje su propio sentido que va desde cualquier expresión artística que puede ser mostrada a través del ciber espacio, hasta construcciones futuristas que sugieren la existencia de hiperrealidades que se configuran a cada paso y que hacen de cada experiencia un mundo en permanente resignificación.

Todo esto es posible y desde la perspectiva de la filosofía cuántica podríamos asegurar que no sólo lo es, sino que ya lo está siendo de forma simultánea y que sólo debemos esperar a que dicha realidad se haga palpable.

Es así como el arte instancia aparentemente intangible se cruza con dimensiones más o menos virtuales tales como el dinero virtual, la billetera

4 Desde aquí también nos replanteamos a la lógica, en este caso a la lógica binaria aristolélica e introduce desde la mirada del tercer incluido a la lógica cuántica que nos habla de simultaneidad.

EMUI_EuroMed University | ISSN 1578-6730 - Nomads. Mediterranean Perspectives | ISSN 1889-7231 (c) 1999, Román Reyes, Fundador y Director · (c) 2016, Progetto Pier Paolo Pasolini 
móvil o las redes sociales expresadas éstas a través de las redes inalámbricas de la telefonía digital y la red de internet que vehiculiza todo lo dicho anteriormente. Acercándonos de este modo al siguiente cuestionamiento:

¿Cómo se puede hablar de sensibilidad humana o incluso de crítica a la existencia o no de dicha sensibilidad, cuando ésta se cruza con la crudeza de la racionalidad virtual?

Lo mismo para cualquier ámbito de la vida en especial para el universo educativo.

¿Cómo enseñar, para qué enseñar, estamos en la era del autoaprendizaje o del aprendizaje colaborativo, como influyen las redes sociales y las plataformas cibernéticas en todo esto?, nos dirigimos hacia una cultura cyborg o ya estamos atravesando la era de la tecnoreligión transhumana? ¿Es el transhumanismo una fe 0 simplemente una manifestación más del miedo a la muerte?

\section{CIBERSOCIEDAD EN PERSPECTIVA TRANSCOMPLEJA}

"La Internet ha sido llamada "Pangea", "alfombra mágica", "Autopista de la Información", "Red de Redes", "megarred", "ser vivo", "nueva realidad", "realidad paralela", "Galaxia", etcétera. Al ciberespacio se le ha denominado "aleph", "alucinación consensuada", "hiperrealidad extendida", "cibersociedad", "cibercapitalismo", "realidad virtual amplificada", etcétera" 5 .

"En el centro de nuestra era tecnológica se halla un grupo fascinante de personas que se autodenominan hackers. No se trata de celebridades televisivas con amplia fama y reconocimiento, pero todos conocen sus logros, que constituyen la nueva base tecnológica de la sociedad emergente: Internet y la Red de redes (lo que en conjunto podríamos llamar la Red), el ordenador personal, así como una parte importante del software utilizado para que todo ello funcione"6

En su desesperado intento por crear cultura, el mono creativo de "Desmond Morris"7(otrora mono desnudo) busca alcanzar un algo que tal

5 Adame Cerón, Miguel Ángel. Redes urbanas y virtuales del capitalismo "glocal". Rebelión.org

6 Himanen, Pekka. La ética del hacker y el espíritu de la era de la información.http://www.educacionenvalores.org/IMG/pdf/pekka.pdf

7 Morris, Desmond. El Mono Desnudo. Ediciones Plaza y Janes. Barcelona España 1968. http://fierasysabandijas.galeon.com/enlaces/libros/mondes.pdf 
vez nunca se ha ido. El hombre hacedor de objetos es reconfigurado por el objeto mismo, este homo tecnológicus no necesita un conocimiento especializado para desarrollar un determinado uso. Es decir, que una persona cualquiera no necesita un doctorado en electrónica para encender un televisor o un radio, mucho menos necesita conocer cuáles son los pasos que sigue un determinado producto industrial-alimenticio (como por ejemplo unas papitas fritas) para poder disfrutar del mismo. En consecuencia, tampoco necesitamos conocer mucho acerca de cómo funciona Internet (técnicamente hablando) y de toda la cultura massmedíatica que esto engloba para ser afectado por ella. Entonces podríamos decir que: ¿La mediatización telemática es el puente entre el Homo Sapiens y el Homo Tecnológicus? O zpor otra parte entre dicha mediación telemática y el homo sapiens hay otras instancias aun claramente no entendidas?

Otra expresión derivada de las anteriores es la de cibernauta, que al conectarla con lo expresado anteriormente, nos sugiere a un tipo de persona que sin ser necesariamente un experto en sistemas informáticos, es capaz de deslizarse por la red e incluso hacer aportaciones a la misma casi sin darse cuenta. Desde esta perspectiva emergen múltiples lecturas que van desde la paranoica conspiracionista hasta la romántica idealista. Todas ellas e incluso las posiciones intermedias, no sólo pueden coexistir sino que de hecho coexisten de forma más o menos armónica en la red de redes.

\section{CIBER PENSAMIENTO}

El universo informático además de haber construido un lenguaje propio (ciber-diccionario), ha traspasado el umbral del pensamiento moderno, conformando de este modo una nueva estructura cognitiva, una nueva forma de aproximarse a lo cotidiano para hipertexturizar el tiempo y desconfigurar el espacio.

Pensar la virtualidad es perderse en y a través de realidades múltiples/paralelas que cohabitan nuestra comprensión y nos hacen viajar sin viajar, mirar sin mirar, sentir sin sentir. $Y$ en consecuencia existir $O$ no existir al mismo tiempo.

Pensar el universo ciber constituye pensarnos a nosotros mismos y preguntarnos constantemente si nuestra realidad, esa instancia densificada en la que vivimos a diario, es real o no y si eso que llamamos irreal (imaginación, sueño, recuerdo, etc. Es real o no. En definitiva nuestro tránsito por dicho universo relacional nos conduce 
irremediablemente a cuestionar la naturaleza de nuestra lógica. ¿Es binaria $\circ$ polivalente nuestra lógica, es binaria o polivalente la realidad sea esta real o virtual? Incluso ¿̇son dichas realidades antes mencionadas una sola, y eso que llamamos real y virtual no son más dos manifestaciones de una misma realidad?

Amador Martos inspirado en los textos de Ken Wilber, en su libro educación cuántica nos plantea la relectura de la filosofía occidental desde un replanteamiento neokantiano que pone en cuestionamiento la separación de la ciencia, la moral y el arte. Esa disyunción nos invita a la integración e incluso a comenzar $a$ ir en un sentido no pensado anteriormente. Martos nos dice:

"La razón ha quedado conmocionada al estrellarse en el estudio de la materia mediante la física cuántica, lo cual ha producido un giro copernicano en la mirada desde el "ver para creer" al "creer para ver", desde el método científico a la fenomenología, desde el racionalismo pragmático al racionalismo espiritual, desde el materialismo científico al misticismo cuántico, desde el neoliberalismo al altermundismo, desde la psicología tradicional a la psicología transpersonal, desde la filosofía tradicional a la filosofía transpersonal, y cómo no, de la educación tradicional a La educación cuántica. Tantos cambios de paradigmas implican un revisionismo humano, vislumbran la necesidad de una renovada epistemología". ${ }^{8}$

\section{RELATIVIDAD Y CUÁNTICA}

Si bien es cierto que a comienzos de siglo con las publicaciones de la teoría general de la relatividad (1913) por Albert Einstein; se generó toda una revolución en el pensamiento global, así como las ideas derivadas de la Física cuántica tales como el principio de incertidumbre de Heisenberg y la idea de cuantos de energía de Max plank. También es cierto que la humanidad aún sigue altamente influenciada por la concepción lineal mecanicista de la ciencia. Es decir, sigue gobernada por una visión predictiva, exacta, cuantificable, comprobable, invariable y casi perfecta. Por lo tanto los seguidores de este paradigma aún creen en la posibilidad de poder predecir con exactitud la gran mayoría de los fenómenos naturales. Sin embargo, en el universo cibernético pareciera que las relaciones espacio-temporales se conformaran de otro modo. En tal sentido, nos movemos sin movernos, estamos y no estamos, y en relación al tiempo resulta totalmente intrascendente el aquí y el ahora, el antes y el después.

8 MARTOS, A. (2017) La Educación Cuántica. Un nuevo paradigma de conocimiento. CreateSpace, compañía de Amazon.com ISBN: 978-84-697-4774-2

EMUI_EuroMed University | ISSN 1578-6730 - Nomads. Mediterranean Perspectives | ISSN 1889-723 (C) 1999, Román Reyes, Fundador y Director · (C) 2016, Progetto Pier Paolo Pasolini 
Martos en relación a la nueva educación nos plantea:

"Es oportuno e importante hacer una diferenciación pedagógica entre las escuelas tradicionales y las escuelas activas. Mientras que en la escuela tradicional prima el aprendizaje memorístico, en la escuela activa se imparte un aprendizaje comprensivo, crítico y multidisciplinar. En la primera, la relación entre maestro y alumno es de autoridad y pasiva recepción de conocimientos, respectivamente. Sin embargo, en la escuela activa, se plantea el aprendizaje a partir de las necesidades e intereses del alumno, siendo el maestro un acompañante participativo en la construcción del conocimiento. En la escuela tradicional se hacen exámenes. Sin embargo, en las escuelas activas, se evalúa el progreso del alumno de manera global, no por área y materias, sino por medio de acuerdo de las normas entre todos, es decir, consenso frente a la actitud represiva de la escuela tradicional".

Así pues una educación cuántica ha de ser una educación más humana aún cuando dicha humanidad sea impartida por artefactos tecnológicos.

\section{LA CIBER LÓGICA Y LA CIBER ÉTICA}

Las Razones que cimentaban el orden y el funcionamiento de nuestro mundo clásico, se han visto perturbadas por una nueva lógica fluctuante, aleatoria, cuasi-caótica y en tal sentido difícilmente predecible. La ciber lógica se halla consustanciada con una nueva lógica llamada lógica difusa o también llamada lógica polivalente en la cual el clásico razonamiento basado en "sí y no" (0 y 1) es atravesado y superado por infinitos espacios intermedios entre las distintas categorías de "si" Y "no". En consecuencia las posibilidades de respuesta ante un determinado problema se multiplican y además de todo esto se hacen inseguras, surge de este modo una nueva concepción del mundo y de las cosas fundada en la incertidumbre. De aquí el principio de incertidumbre de Heisenberg el cual nos abre los ojos ante la perspectiva de un universo azaroso y multifactorial.

En torno a la ciber ética, Denise Najmanovich nos aproxima cuando nos habla de la multidimencionalidad de la experiencia, en la cual las experiencias interactivas (cibernéticas) se suman a las experiencias de la vida de cada quien y arma una especie de "Pastiche" el cual es posteriormente interpretado por nuestra neocorteza, generando de este modo una visión (cosmovisión) del mundo, la cual es propia de cada

9 Idem

EMUI_EuroMed University | ISSN 1578-6730 · Nomads. Mediterranean Perspectives | ISSN 1889-7231 (C) 1999, Román Reyes, Fundador y Director · (C) 2016, Progetto Pier Paolo Pasolini 
individuo y es aquí donde el loco, el poeta, el asesino, el sicópata, el amante, el estudiante, el morboso, el religioso, etc., se encuentran en un ciber café uno al lado del otro y se dan la mano, y en otra visión aún más relativa de la ética nos encontramos con un mismo sujeto transformándose en cada uno de los estereotipos antes citados a través del pestañeo de un clic. ¿̇Hasta dónde está visón de la ética puede ayudarnos en y hacia la construcción de un mundo nuevo que promueva la paz y la cooperación? Una cosa es segura, no será a través de los video-juegos violentos que practican nuestros niños hoy.

Una mirada más espiritual de la ética la observamos al momento de activar nuestros sentidos internos, desde esta perspectiva nuestro yo interno, yo superior se convierte en el guía de nuestros procesos y deja de lado el engaño y/o ilusión que nos transmiten nuestros sentidos. De allí la expresión planteada por Martos que dice: Creer para ver. Sin embargo dicho creer para ver no es una instancia mágica, al contrario es una dimensión extremadamente lógica y coherente de allí que la clave para activar nuestros sentidos internos, no es la ilusión y la fantasía sino la coherencia/congruencia en nuestro sentir/pensar/hacer cotidiano.

\section{HOMO-MAQUINUS}

No necesito recordarles a Julio Verne para decirles que la ciencia ficción de hoy puede ser ciencia real en el futuro y tampoco necesito recordarles a las telenovelas, las series televisivas, las películas y los comics, los cuales en el principio se nutrían de la vida diaria para ser creados, y ahora son ellos los que nos crean y nos recrean. Recordemos a Eduardo Liendo en su incansable lucha contra El Mago de la cara de Vidrio:

"Sólo una vez la suerte del mago estuvo en mis manos, pero ignoro si entonces actué como un magnánimo adversario o como un idiota redomado"10

"Dadme apenas un soplo de aliento, y esta manos firmes multiplicaran su fuerza y lo lanzaran por la ventana. ;Os llamo al combate frontal contra el mago de la cara de vidrio! Seguidme!...""l

¿Hacia dónde vamos?, ¿Que distancia existe actualmente entre el hombre y la máquina? ¿Estamos cerca de hacer un hombre-máquina? Ó por el contrario ¿̇Estamos más próximos a construir una máquina

10 LIENDO, E. (1973) El mago de la cara de vidrio. Monte Ávila editores, latinoamericana. 11 Idem

EMUI_EuroMed University | ISSN 1578-6730 · Nomads. Mediterranean Perspectives | ISSN 1889-7231 (C) 1999, Román Reyes, Fundador y Director · (C) 2016, Progetto Pier Paolo Pasolini 


\section{humana? "Mañana voy a comprarme un robot que me enseñe a ser persona"12}

No nos resistimos a la idea de que personas con daños neurológicos incapacitantes o con discapacidades motoras, utilicen medios tecnológicos para hacer más llevaderas sus vidas pero de allí a convertirnos en ciborgs y sobre todo ciborgs para la guerra, es un debate que tenemos que dar.

Términos como: Biónica, Biorrobótica, Biotelemática, Inteligencia Artificial, vida artificial, entre otros, nos hablan de los intentos que vienen haciendo los seres humanos para darle respuesta a las preguntas arriba planteadas. Pero más allá de los posibles avances y logros en estos interesantes campos del conocimiento. El hombre común, "el homo cotidianus"13 se pregunta ¿̇a dónde nos conducirá todo este desarrollo ${ }^{14}$, y surge la angustia tecnológica que no es más que el temor irrefrenable hacia cosas no existentes, pero que en un futuro podrían existir. ¿̇s realmente valido asustarse así', recordemos los marcianos de Orson Wells 15 , y respondamos: ¿Fueron reales o no? pues para aquellas personas que murieron sí que lo fueron y de aquí el surgimiento de otra frase: "realidad virtual", la cual también pertenece al ciberdiccionerio. Y es que hoy día con el surgimiento de toda esta nueva lógica

12 FERNÁNDEZ, O. (2012) Espiralario. Editorial el perro y la Rana. Caracas Venezuela. FERNÁNDEZ, O. (2012) Entre el Cristal y las Nubes. Ensayo sobre Biología Filosófica. Editorial el perro y la Rana. Caracas. Venezuela.

13 Este homo cotidianus nos recuerda a la serie de dibujos animados, "Los supersónicos", que necesita un doctor especialista en dedos, ya que es ese el único órgano que utilizan por presionar tantos botones en una sociedad del futuro que nunca llega. O como pasa en viaje a las estrellas, cuando el Dr. Macoy viaja al pasado (síglo $X X)$ e ingresa a un hospital y le da a un paciente una pastilla que le hace crecer un riñón nuevo. No sólo es ficción todo lo dicho arriba, es también una promesa de la modernidad que nunca satisfizo.

14 Cabría preguntarnos: ¿¿Desarrollo entendido en base a qué términos? Porque si es el desarrollo en base al crecimiento económico que a su vez se sustenta en la destrucción del planeta? entonces ese desarrollo no es el nuestro.

15 En relación a la paranoia que desató Wells, podríamos citar hoy día el caso de los llamados prepers y el caso de aquellas personas que por ejemplo entran en crisis si pierden el teléfono celular, el primer caso forma parte de to que podríamos llamar una patología social derivada de los países mal llamados desarrollados y que así como su sistema los lleva a fabricar armas para promover conflictos en el mundo, también a lo interno las consigues como caramelos y produce casos como los que llevaron a niños a masacrar a otros niños. Los prepers son paranoicos que creen que el mundo se va a acabar y deben tener todos los insumos necesarios para sobrevivir en caso por ejemplo de una catástrofe nuclear. Y por último nombramos el caso de la adicción a los celulares pero puede ser a cualquier artefacto tecnológico, que así como cualquier droga, el consumismo mundial crea dependencia y por lo tanto induce a más y más consumo.

EMUI_EuroMed University | ISSN 1578-6730 - Nomads. Mediterranean Perspectives | ISSN 1889-723 (c) 1999, Román Reyes, Fundador y Director · (c) 2016, Progetto Pier Paolo Pasolini 
(ciberlógica) la concepción de realidad y en consecuencia la de verdad deben ser rediscutidas.

\section{CIBER EPISTEMOLOGÍA}

"La gente critica con frecuencia la falta de nexos entre lo que se dice y lo que se hace, entre lo que se piensa y lo que se practica. Ello quiere decir que uno espera una cierta coherencia entre el pensar y el hacer. Pero el asunto es aún más intrincado: hay una correspondencia entre las mentalidades y las prácticas aún sin que nos lo propongamos. Esto significa que-la eficacia de las ideas, las creencias, las convicciones o los prejuicios es mucho más fuerte de lo que solemos imaginar. Podríamos concluir con esta tesis sencilla: la gente hace lo que hace según el paquete de ideas que tiene en su cabeza. Ello vale para todas las esfera de la vida. En cualquier espacio encontraremos a las personas haciendo esto o aquello, realizando unas prácticas y dejando de realizar otras justamente en atención a su mentalidad, a sus creencias, a su nivel intelectual, a las ideas que tienen en mente". (Rigoberto Lanz). ${ }^{16}$

Al pensar sobre lo pensado, se pueden dar dos fenómenos: En el primero se parte de un punto, y se regresa siempre al mismo lugar. Y en el segundo, se parte y se retorna a un mismo sitio en apariencia pero distinto en esencia; diríamos pues que hablamos de un circulo y de un espiral del pensamiento respectivamente.

- El círculo y la espiral no son opuestos; por el contrario son complejamente complementarios.

- El circulo se espiraliza en el momento de establecer vínculos (pautas- Patterns) que se interconectan entre sí.

- Y la espiral se hace círculo cuando decide volver a lo ya pensado con la experiencia espirílica.

- Curiosamente el retorno al círculo proveniente de la espiral, lo separa de su condición de círculo y lo convierte en un puente entre ambas estructuras de pensamiento.

- $\quad$ En el dominio círculo-espirílico la distancia se presenta en medio de una existencia paradojal. Pues alejarse es acercarse y viceversa.

16 Lanz, Rigoberto. ¿Cuál ciencia? Fundación para el desarrollo de la ciencia y tecnología del estado Zulia. http://www.fundacitezulia.gov.ve/fnd/index.php?option=com_content\&view=article\&id=49:icual-

ciencia\&catid=58: articulos-de-interes\&ltemid $=2$

EMUI_EuroMed University | ISSN 1578-6730 · Nomads. Mediterranean Perspectives | ISSN 1889-7231 (C) 1999, Román Reyes, Fundador y Director - (C) 2016, Progetto Pier Paolo Pasolini 
- $\quad$ Dentro de la paradoja de la distancia círculo-espirílica alejarse de una idea, es acercarse a ella desde un lugar distinto; desde otra perspectiva; no es abandonar, es retornar.

- $\quad$ ¿Es una pérdida de tiempo pensar lo ya pensado?

- $\quad \dot{2}$ Tiene el pensamiento realmente un comienzo y un fin?

- $\quad$ ¿Qué o quién decide que algo o alguien ya no merece ser pensado?

- $\quad$ ¿Es la lógica circulo-espirílica un intento de anulación de la lógica Aristotélica de separación de los opuestos?

- Pensar acerca de la condición de espacio-tiempo del pensamiento, no tiene ni inicio, ni fin, ni lugar, ni distancia.

\section{CIBERIDEOLOGÍA}

"Para Engels, a Dialética da Natureza consiste em uma visão de mundo ou perspectiva geral cuja utilidade terá que ser mostrada a posteriori, na medida em que frutificar em avanços científicos concretos." 17

Hablar del fenómeno ideológico presente en y a través del ciberpensamiento, es todo un reto, sin embargo creo que existen algunos elementos que sugieren que dicho actuar y pensar no son inocuos tal y como algunos entendidos en estos temas pretenden sugerir. En tal sentido podemos comenzar por decir que en este universo de relaciones, actúan intenciones tan capitalistas como las de Microsoft y Google, que al mezclarse interactivamente se cruzan con Wikipedia y el sistema operativo LINUS, por otro lado tenemos a las redes sociales que por un lado generan dividendos asombrosos para sus creadores y que por otra parte han promovido una verdadera revolución en las relaciones humanas. Pero si a todo esto le añadimos las denuncias de Julian Paul Assange y Edward Snowden, todo se complejiza, Pero más allá tenemos la experiencia de movimientos políticos inspirados en el ciberespacio:

"El 2009 marca una etapa importante en los avances y los cambios en ámbito de los derechos y de la comunicación: con las elecciones europeas del 7 de Junio de 2009, en Suecia el Pirat Partiet ("Partido Pirata", nacido en Suecia en 2006) consigue ser el primer partido votado por los menores de 30 años, y logra también tener un escaño en el parlamento Europeo. Actualmente (a finales de 2009) en varios países del

17 Para Engels la Dialéctica de la naturaleza consiste en una visión del mundo o una perspectiva general, cuya utilidad tendrá que ser mostrada a posteriori, en la medida en que prosperen los avances científicos concretos. Kinouchi, Osame. Um Precursor das Ciencias da Complexidade no Século XIX. Faculdade de Filosofia, Ciencias e Letras de Ribeirao Preto Universidade de Sao Paulo. Av. Bandeirantes, 3900, CEP 14040-901, Ribeirao Preto, SP, Brazil. February 2, 2008.

EMUI_EuroMed University | ISSN 1578-6730 - Nomads. Mediterranean Perspectives | ISSN 1889-723 (C) 1999, Román Reyes, Fundador y Director - (C) 2016, Progetto Pier Paolo Pasolini 
mundo han nacido homónimos partidos, que comparten su filosofía. En España el recién Partido Pirata se está organizando para poder participar a las elecciones Europeas de 2011 , y en varios países iberoamericanos ya se han ido difundiendo formaciones políticas con el mismo programa, en defensa de los derechos de los ciudadanos, considerados también como "navegantes" de la red, que incluyen dentro de los derechos humanos el derecho de libre información, intercambio de datos, y libre expresión"18

Hay quienes incluso hablan de política 2.0, y creen que a través de los medios cibernéticos se está gestando una verdadera revolución político/social.

Así pues, tenemos que la emergencia de una ciber-ideología en el mundo ya no es un relato de ciencia ficción. Solo un aspecto queda claro, y es que esta nueva ideología no se puede medir con el mismo metro de las ideologías del pasado, pues en algunos aspectos las incluye y en otros se separa radicalmente marcando una clara distancia tanto ideológica como epistemológica. Es éste, el surgimiento de una nueva forma de ver y pensar la política, que no será por supuesto la única ni la última. Y si a todo lo antes dicho le sumamos la idea de una nueva concepción de desarrollo leída desde y a través del ciberpensamiento, esta sería una idea que trascendería las nociones de espacio y tiempo y presentaría al igual que la noción de ecodesarrollo, la necesidad de considerar al planeta todo como el eje de las transformaciones, y ya no se hablaría más de territorio, tal vez le agregaríamos el prefijo eco, o en el caso de la ciberideología denotaríamos al término ciberdesarrollo'19, este al igual que el ecodesarrollo20 iría más allá de la concepción econométrica de desarrollo que concibe al mismo como mero crecimiento económico e incluiría en esta dimensión no sólo a los seres vivos y sus interacciones con el entorno, sino que además incluiría a las interacciones antes descritas con las interacciones hombre/máquina y máquina/máquina, regidos por una ciberlógica que pone en juego a la inteligencia artificial, vida artificial y espacios intermedios como los ciborgs y los memes.

18 ADDOLORATO, A. Generación tuteo. Red(es), arte, sociedad. Cultura española siglo XXI. CUEM soc. Coop. Milano 2009 Italia.

19 Este ecodesarrollo que aquí proponemos se inscribe en y a través de la mirada del ecoparadigma propuesto por Fritjof Capra y el cual deriva a su vez de la ecología de la mente o ecología de las ideas de Gregory Bateson.

20 Por otra parte el ciberdesarrollo debe conjugar una mirada que trascienda la noción de territorio y que entienda que el desarrollo desde la perspectiva ciber debe conducirnos a una nueva cartografía ya no del espacio sino de las ideas y en especial de los consensos.

EMUI_EuroMed University | ISSN 1578-6730 - Nomads. Mediterranean Perspectives | ISSN 1889-723 (C) 1999, Román Reyes, Fundador y Director · (C) 2016, Progetto Pier Paolo Pasolini 
Otra mirada nos pone a ver las relaciones espacio temporales derivadas de la interacción entre los lugares y los no lugares, tal como lo define Miguel Ángel Adame Cerón en un artículo de nombre: Redes urbanas y virtuales del capitalismo "glocal":

"La fluidez circulatoria-comunicativa de la sociedad capitalista global convierte cada sujeto, conexión, vía, ordenador, calle, plaza, edificación, espacio público o privado en nodo y eje partícipe de las tensiones e hibridaciones micro-macro reticulares, espacios de fijosespacios de flujos, lugares-no lugares, etcétera"21.

De esta forma nos enredamos en las relaciones constitutivas de la vida que de una $u$ otra forma se configuran en una lectura de la vida. También nos dice Miguel Ángel Adame Cerón en relación a la red de redes:

"La cibercultura se presenta ahora como globalizadora, mejor dicho glocalizadora, es decir, con tendencias abarcadoras de los ámbitos más importantes -globales y locales- de lo sociocultural (Adame, 2004b:307407); específicamente lo económico, lo militar, lo científico-tecnológico, lo político, lo doméstico y la vida cotidiana, así como lo ideológico, lo educativo y el ámbito de las diversiones-ocio, los juegos y el placer (Adame, 2004a:45-64). Jorge Alor definió la cibercultura como: "todo lo creado por el hombre en su devenir constante de procesos digitales individuales y colectivos que están en constante renovación y que está cambiando los hábitos, el cuerpo-mente, las relaciones interpersonales y las relaciones con las máquinas" (De Rivera, 2000:60). Pero en realidad hay más: lo cibercultural se presenta actualmente como todos los procesos científico-tecnológicos donde intervienen los conocimientos, los imaginarios y las múltiples aplicaciones mentales y de mecanismos y artefactos de índole cibernética lo sea de la ciencia que se erigió después de la segunda guerra mundial -junto con la sistemática- como la más intercomunicadora e integradora basada en el control y la comunicación con base en dispositivos autorregúladores y autoadaptables) para transformar y modelar todos los ámbitos de la vida socio-cultural posmoderna e hipermoderna. El engendro tecnológico más espectacular, exponencial y de vanguardía que da sentido y dirección, que modela y retroalimenta cibersocioculturalmente a la macrotelaraña del capital glocal desde este siglo XXI es el denominado ciberespacio que tiene su Red de redes central denominada Internet"22.

21 Adame Cerón, Miguel Ángel. Redes urbanas y virtuales del capitalismo "glocal". Rebelión.org

22ldem

EMUI_EuroMed University | ISSN 1578-6730 · Nomads. Mediterranean Perspectives | ISSN 1889-7231 (c) 1999, Román Reyes, Fundador y Director · (c) 2016, Progetto Pier Paolo Pasolini 
De esta forma lo ideológico se entreteje en la telaraña de relaciones glocales que hacemos y a la vez nos confunde a través de la envolvente y engañosa espiral que llamamos vida.

Otros temas que por lo menos no pueden dejar de mencionarse son el ciberterrorismo y el ciberespionaje. Los órganos de inteligencia de los países poderosos, han desarrollado tremendos sistemas informáticos que han roto la sensación de privacidad que en otrora creíamos poseer. Así pues, no podemos escapar de esta red que nos enreda.

En relación a lo social y la educación cuántica Martos nos dice:

"La educación cuántica y el movimiento altermundista están inherentemente relacionados: la primera propugna una evolución holística del "yo" hacia el "nosotros" mediante la fuerza del saber y del amor $y$, el segundo, evidencia una incipiente conciencia social del "nosotros" como revulsivo a la egolatría plutocrática, quien está en el origen de la actual estafa a la humanidad eufemísticamente llamada crisis"23.

El ser humano, animal de costumbre ha hecho de su interacción con lo tecnológico (lo cibernético), un continum de interretroacciones que lo encarcelan en medio de un circuito de artefactos que a la vez lo hacen dependiente de sus aplicaciones y modalidades. Es así como la red de redes se vuelve toda una trampa consumista que nos seduce y que luego puede llegar a destruirnos mientras nos une o nos separa de los demás seres humanos.

\section{CONSCIENCIA CYBORG}

"¿Cuándo se convierte en consciencia un simulador perceptual?"

(Yo Robot, Isaac Asimov)

En obras de ciencia ficción como yo robot, hombre bicentenario, y ex máquina, se debate sobre la posibilidad de generar por vía artificial la consciencia humana. Por otra parte se sugiere la posibilidad de que la vida artificial supere de algún modo a la vida natural a través de una condición más sublime... la consciencia humana.

23 MARTOS, A. (2017) La Educación Cuántica. Un nuevo paradigma de conocimiento. CreateSpace, compañía de Amazon.com ISBN: 978-84-697-4774-2 
Pero es que no todos los humanos son conscientes de su consciencia, la mayoría de la humanidad anda por el mundo desconociendo su propia naturaleza. Desde esa perspectiva ser humano, es ser artificial. Y si ese es el punto de partida de la inteligencia artificial, estaríamos hablando de la artificialidad de la artificialidad.

Para muchos la consciencia humana constituye la cualidad que nos diferencia de los demás organismos y máquinas. Pero ¿Qué es la consciencia?

Para Ken Wilber por ejemplo la consciencia la define nuestra naturaleza perceptiva de la realidad la cual a su vez se encuentra limitada por barreras que nosotros mismos creamos, en tal sentido nos dice:

"... creamos una persistente alienación de nosotros mismos, de los otros y del mundo, al fracturar nuestra experiencia presente en diferentes partes separadas por fronteras. Efectuamos una división artificial en comportamientos de lo que percibimos: sujeto frente a objeto, vida frente a muerte, mente y cuerpo, dentro y fuera, razón e instinto, y así recurrimos a un divorcio causante de que unas experiencias interfieran con otras y exista un enfrentamiento entre distintos aspectos de la vida". 24 Desde esta perspectiva emergen por lo menos dos hipótesis, primera: si la realidad que conocemos es ilusión entonces la realidad virtual y en consecuencia, eso que podríamos denominar realidad alterna sería una ilusión alterna o la artificialidad de lo que ya es artificial.

Y segunda: si la conciencia real es esa que aún no conocemos: ¿ Cómo deberíamos hacer para simularla a partir de lo que sí conocemos, aun cuando esta sea parcial?

Si podemos diferenciar con claridad lo real de lo no real, una inteligencia artificial que tenga clara esta situación podría conducirnos no a una ilusión de la ilusión sino a un despertar de la conciencia. Dicho despertar de la consciencia podría darse en un cuerpo humano o en uno artificial. Y así podríamos repetir: "Mañana me compraré un robot que me enseñe a ser persona?25

Intentemos dar respuesta a la pregunta que inicia esta sección:

"¿Cuándo se convierte en consciencia un simulador perceptual?" una respuesta intuitiva podría ser: cuando emerja la percepción simultánea multinivel (intuición)

Pero żqué es la consciencia y qué es la intuición?

24 WILBER, K. La conciencia sin fronteras. Aproximaciones de oriente y occidente al crecimiento personal. (2011) Editorial Kairós.

25 FERNÁNDEZ, O. (2012) Espiralario. Editorial el perro y la Rana. Caracas Venezuela.

EMUI_EuroMed University | ISSN 1578-6730 - Nomads. Mediterranean Perspectives | ISSN 1889-7231 (C) 1999, Román Reyes, Fundador y Director · (C) 2016, Progetto Pier Paolo Pasolini 
En yo robot vemos que Asimov caracteriza lo que según él es la consciencia sugiriendo que los secretos, los sueños, las emociones, los pensamientos, la personalidad, el humor, la creatividad, entre otros constituyen instancias propias de la consciencia humana.

Por otra parte Ken Wilber nos habla de los niveles o espectros de la consciencia sugiriéndonos que dichos niveles en realidad no existen y que somos nosotros los que de forma inconsciente creamos dichos límites y/o fronteras. Él plantea: el nivel subconsciente (ego-sombra), cuerpomente (centauro), inconsciente colectivo (transpersonal), y el nivel de la unidad. Éste último nivel según Wilber en realidad es el único que existe y es el único desde el cual deberíamos responder a la pregunta: ¿Quién soy yo?

Volviendo a la reflexión sobre la vida artificial o la artificialidad de la vida, podríamos decir que el inicio del desarrollo de la consciencia de una máquina iniciaría cuando ésta sea capaz de preguntarse a sí misma: ¿Quién o qué soy yo?

Volvamos un poco más a lo que Wilber define como el espectro de la consciencia.

Wilber comienza cuestionando la lógica aristotélica diciendo:

"Obsérvese que todas las dimensiones espaciales y direccionales son opuestas: arriba y abajo, se oponen, como dentro y fuera, alto y bajo, largo y corto, norte y sur, grande y pequeño, aquí y allá, cima y fondo, izquierda y derecha. $Y$ todas las cosas que consideramos serias e importantes son un polo de un par de opuestos: bien y mal, vida y muerte, placer y dolor, Dios y Satán, libertado y servidumbre."26

"También nuestros valores sociales y estéticos son siempre algo que se da en función de opuestos: éxito y fracaso, bello y feo, fuerte y débil, inteligente y estúpido. Incluso nuestras abstracciones supremas se fundan en oposiciones. La lógica por ejemplo, se ocupa de lo verdadero y lo falso, la epistemología de la apariencia y de la realidad, la ontología del ser y el no ser. Parece que nuestro mundo es una impresionante colección de opuestos" 27

Desde aquí observamos que la posibilidad de transformar nuestra consciencia parte por cuestionarnos el sentido de lo que siempre hemos considerado como única verdad. En este caso en particular, la lógica.

Hablemos del nivel ego-sombra: En este nivel Wilber se refiere al nivel del subconsciente y el cómo éste basado en esa relación de los opuestos

26 WILBER, K. La conciencia sin fronteras. Aproximaciones de oriente y occidente al crecimiento personal. (2011) Editorial Kairós.

27 Idem

EMUI_EuroMed University | ISSN 1578-6730 · Nomads. Mediterranean Perspectives | ISSN 1889-7231 (C) 1999, Román Reyes, Fundador y Director - (C) 2016, Progetto Pier Paolo Pasolini 
nos controla sin darnos cuenta. Wilber citando a Freud nos dice: «Toda la teoría psicoanalítica se asienta, en efecto, en la percepción de la resistencia que ejerce el paciente cuando intentamos hacer que tome conciencia de su inconsciente» ${ }^{28}$. Este primer nivel constituye la base de todos los demás y según Wilber, al superar este, instantáneamente se superan todos los demás ya que los anteriores caen como un castillo de naipes. Es en este nivel donde hallamos el origen de nuestros más terribles miedos y de nuestras dolencias físicas más fuertes. Ya resistencia a mirarnos como totalidad, es la que genera toda la perversión humana. El ying y el yang son una unidad y como tal debemos aprender a percibirla. Aquí el terapeuta se centra en la "búsqueda de bloqueos que puedan afectar el flujo continuo del pensamiento y las emociones.

El siguiente nivel el del centauro o nivel cuerpo-mente: Es aquel que nos pone en evidencia nuestra relación con nuestro cuerpo físico. Vivimos en un mundo en el que la inmediatez, la competitividad y la superficialidad de las cosas nos hacen víctimas de nosotros mismos. La relación cuerpo y mente queda escindida. De allí que somos capaces de percibir una dolencia física ya cuando estamos a punto de morir. El alejamiento de nuestro cuerpo es el alejamiento de nosotros mismos. Aquí nos dice Wilber: "El terapeuta estará alerta, pero no a la aparición de bloqueos en el pensamiento, sino a cualquier huida de la percepción del presente hacia el pensamiento"29.

En el siguiente nivel, nivel del inconsciente colectivo o nivel transpersonal, partiendo de los estudios de Carl Gustav Jung, Wilber nos introduce en ese nivel que trasciende lo personal, que nos habla de un yo superior, en términos cuánticos podríamos decir que sería establecer conexión con ese o esos seres que se hallan en dimensiones superiores a la nuestra y que no son otra cosa que nosotros mismos con niveles de consciencia más elevados. Wilber nos dice: "Vivir mitológicamente la vida significa, más bien, empezar a aprender lo trascendente, sentirlo vivir en nosotros mismos, en nuestra vida, el trabajo, los amigos, y el entorno. La mitología nos abre, precisamente, a ese mundo de trascendencia. Como decía Coomaraswamy, la frase: hace muchísimo tiempo... con la que suelen comenzar los cuentos de hadas, significa en realidad, más allá del tiempo... , y el cuento que sigue pertenece a un mundo donde el espacio y el tiempo se suspenden temporalmente, donde el juego es la regla suprema y puede suceder cualquier cosa"30

Es esta percepción de la realidad de la totalidad, más real que eso que habitualmente denominamos realidad, mediada ésta por la lógica

28 Ibidem

29 Ibidem

30 Ibidem

EMUI_EuroMed University | ISSN 1578-6730 · Nomads. Mediterranean Perspectives | ISSN 1889-7231 (C) 1999, Román Reyes, Fundador y Director · (C) 2016, Progetto Pier Paolo Pasolini 
aristotélica de los opuestos. No se trata pues alejarse del mundo o negar la existencia de este, se trata de percibir desde otra perspectiva, que sin negar lo anterior reconoce que existe un más allá de lo conocido hasta ahora. Esto es comparable a lo que ocurre con la mecánica newtoniana y la mecánica cuántica, ambas son diferentes pero coexisten, sólo debemos saber cuándo actúa la una o la otra.

Y el último y no por ello el menos importante es el nivel de la unidad, recordando lo que decía al inicio de esta sección, el nivel de unidad constituye realmente el único y verdadero nivel, es aquel que trasciende todos los anteriores y el que nos habla de lo que realmente somos, más allá de aquellas fronteras que nosotros mismos nos hemos creado. Wilber nos dice que: "... realidad es lo que no tiene fronteras..." 31

También nos dice que dicha consciencia no hay que buscarla, pues el simple hecho de buscarla sugiere que existe un antes y un después y dicha consciencia existe sólo en el presente. Pretender otra cosa sería negar el presente. En consecuencia la unidad, el único nivel, la única realidad sólo es posible alcanzarla desde un cambio de percepción en el aquí y en el ahora. En relación a esto Martos nos dice: "Como escribió Alan Watts: "No hay más que la experiencia. ¡No hay nada ni nadie que tenga la experiencia de la experiencia! No se siente el sentimiento ni se piensan pensamientos, de la misma manera que no se oye el oír, no se ve la visión ni se huele el olfato"32.

Y Wilber nos dice: "Como dijo Aldous Huxley: "El eterno ahora es una conciencials. Y tal como nos referimos a ella, una conciencia de unidad"33.

Chuang Tse: «Si no hay otro, no habrá uno. Si no hay uno, no habrá quién establezca distinciones» ${ }^{34}$.

Volviendo a nuestra reflexión inicial sobre la posibilidad de la existencia de una consciencia a través de la inteligencia artificial, quien propugne dicha búsqueda deberá ser uno o grupo de místicos cuánticos que tengan clara la trascendencia desde la consciencia de unidad.

Transcomplejidad, transracionalidad, y transdisciplinariedad. No son más que expresiones, manifestaciones de una misma realidad. Es decir; la totalidad. La unidad. En tal sentido la consciencia de unidad planteada por Wilber, es al mismo tiempo consciencia de transcomplejidad,

31 Ibidem

32 MARTOS, A. (2017) La Educación Cuántica. Un nuevo paradigma de conocimiento. CreateSpace, compañía de Amazon.com ISBN: 978-84-697-4774-2

33 WILBER, K. La conciencia sin fronteras. Aproximaciones de oriente y occidente al crecimiento personal. (2011) Editorial Kairós.

34 Idem

EMUI_EuroMed University | ISSN 1578-6730 · Nomads. Mediterranean Perspectives | ISSN 1889-7231 (C) 1999, Román Reyes, Fundador y Director - (C) 2016, Progetto Pier Paolo Pasolini 
transracionalidad y transdisciplinariedad. Si las tres nos hablan de totalidad no podría decir que son instancias diferentes pero tampoco que son la misma cosa. Diríamos pues que se trata de distintos ángulos de la misma realidad.

\section{HACIA LA EDUCACIÓN CUÁNTICA}

La propuesta de la educación cuántica nos sumerge en una realidad paralela que no sólo presenta lo tecnológico como una instancia distante y fría sino que además nos dice que siendo éste un universo atravesado también por lo humano, el mismo adquiere una connotación de sensibilidad, obtiene en consecuencia un corpus y un alma que lo hace no sólo poseedor de una dimensión material sino además de una inmaterial la cual a través de la virtualidad se hace simultánea ante nosotros de allí que lo tangible y lo intangible nos toca al mismo tiempo. Hablamos pues de simultaneidad. Dicha condición dual es lo que somos. No somos sólo materia, no sólo somos antimateria, no somos ciencia solamente, no somos espiritualidad solamente, somos la coexistencia de ambas atravesadas por multidimensiones que nos muestran otra forma de percibir, de percibirnos. Porque en definitiva mirar hacia afvera es en realidad mirar hacia dentro y viceversa. Eso sí la mirada debe ser sin barreras sin límites o como diría Ken Wilber: sin fronteras en el espectro de la consciencia.

Es por ello que la educación aquí se constituye en una educación de conocimiento, entendimiento y amor. Siendo el amor la sumatoria armónica de las dos anteriores.

La búsqueda de la armonía es la búsqueda del sentido de nosotros mismos. Y esta búsqueda sólo puede adquirir un sentido trascendente sí y sólo sí, somos capaces de vivir de forma activa y autocrítica en el eterno presente. No se trata de vivir de las rememoraciones de lo que no pude lograr o de los anhelos de lo que deseo alcanzar, tampoco se trata de vivir en una nube de meditación y/o reflexión espiritual que nos aleja de la cotidianidad. Se trata de hacer de cada instante, cada segundo de nuestras vidas en el aquí y el ahora, una permanente acción reflexiva, no cuestionadora de nosotros mismos sino una instancia serena desde y/o a través de la cual, nosotros ya no seamos instrumentos de nuestras emociones, de nuestros pensamientos, de nuestros deseos, de nuestro cuerpo, de nuestra mente, de nuestro tiempo, sino que seamos observadores transpersonales de nuestra propia realidad. Así, sólo así podremos trascender los límites de la consciencia los cuales por demás nunca existieron. Ese sería el objetivo fundamental de la educación cuántica, mostrar el camino de la liberación a través de la integración

EMUI_EuroMed University | ISSN 1578-6730 - Nomads. Mediterranean Perspectives | ISSN 1889-723 (C) 1999, Román Reyes, Fundador y Director - (C) 2016, Progetto Pier Paolo Pasolini 
ciencia/arte/espiritualidad/ética. En todos los procesos formativos en todo nivel y edad.

\section{REFERENCIAS}

ADAME, M. Redes urbanas y virtuales del capitalismo "glocal". Rebelión.org

ADDOLORATO, A. Generación tuteo. Red(es), arte, sociedad. Cultura española siglo XXI. CUEM soc. Coop. Milano 2009 Italia.

FERNÁNDEZ, O. (2012) Espiralario. Editorial el perro y la Rana. Caracas Venezuela.

FERNÁNDEZ, O. (2012) Entre el Cristal y las Nubes. Ensayo sobre Biología Filosófica. Editorial el perro y la Rana. Caracas. Venezuela.

HIMANEN, P. La ética del hacker y el espíritu de la era de la información. http://www.educacionenvalores.org/IMG/pdf/pekka.pdf

Kinouchi, O. Um Precursor das Ciencias da Complexidade no Século XIX. Faculdade de Filosofia, Ciencias e Letras de Ribeirao Preto Universidade de Sao Paulo. Av. Bandeirantes, 3900, CEP 14040-901, Ribeirao Preto, SP, Brazil. February 2, 2008.

MARTOS, A. (2017) La Educación Cuántica. Un nuevo paradigma de conocimiento. CreateSpace, compañía de Amazon.com ISBN: 978-84697-4774-2

MORRIS, D. El Mono Desnudo. Ediciones Plaza y Janes. Barcelona España 1968. http://fierasysabandijas.galeon.com/enlaces/libros/mondes.pdf

LANZ, R. ¿Cuál ciencia? Fundación para el desarrollo de la ciencia y tecnología del estado Zulia. http://www.fundacitezulia.gov.ve/fnd/index.php? option=com_content\&view=article\&id=49:icu al-ciencia\&catid=58:articulos-de-interes\&ltemid $=2$

Leal Larrarte, S. A. (2019). Poshumanismo y poder en el cómic de CF latinoamericano, casos: Inca (Chile), 1874 (México), Condiciones Extremas (Colombia) y Ficcionario (Argentina). En Paakat: Revista de Tecnología y Sociedad [a publicarse en marzo].

LIENDO, E. (1973) El mago de la cara de vidrio. Monte Ávila editores, latinoamericana.

WILBER, K. La conciencia sin fronteras. Aproximaciones de oriente y occidente al crecimiento personal. (2011) Editorial Kairós. 